\title{
How does a non-ionic hydrophobically modified telechelic polymer interact with a non-ionic vesicle? Rheological aspects
}

\author{
Tiago dos Santos ${ }^{\mathrm{a}, *}$, Bruno Medronho ${ }^{\mathrm{a}}$, Filipe E. Antunes ${ }^{\mathrm{a}}$, \\ Björn Lindman $^{\mathrm{a}, \mathrm{b}}$, Maria Miguel ${ }^{\mathrm{a}}$ \\ a Department of Chemistry, University of Coimbra, 3004-535 Coimbra, Portugal \\ ${ }^{\mathrm{b}}$ Physical Chemistry 1, Center for Chemistry and Chemical Engineering, Lund University, POB 124, 22100 Lund, Sweden \\ Received 12 February 2007; accepted 25 March 2007 \\ Available online 30 March 2007
}

\begin{abstract}
The association between hydrophobically modified polyethylene glycol, HM-PEG, and non-ionic vesicles of tetraethylene glycol monododecyl ether, $\mathrm{C}_{12} \mathrm{E}_{4}$, was investigated. HM-PEG is in a triblock form, with an alkyl chain attached to each hydrophilic polymer-end. Such polymer structure is denoted as telechelic. The vesicle average radius was measured by self-diffusion measurements. The system exhibits both a monophasic gel and biphasic regions. The monophasic region was characterized from a rheological point of view. We argue that the gel formation is due to the presence of polymer crosslinks between different surfactant aggregates, once the polymer's hydrophobic moieties may adsorb into the vesicle bilayer. This association is strongly concentration dependent which is reflected in the monotonic increase of the storage modulus, relaxation time and shear viscosity with the addition of surfactant and/or polymer.
\end{abstract}

(C) 2007 Elsevier B.V. All rights reserved.

Keywords: Gels; Hydrophobically modified polymers; Non-ionic surfactants; Rheology; Self-diffusion

\section{Introduction}

Hydrophobically associating water-soluble polymers belong to a class of complex systems recently attracting great interest, mainly due to their self-aggregation properties, thickening, and stabilization. They show amphiphilic behaviour, comprising a water-soluble block and oil-soluble blocks chemically attached to the polymer hydrophilic backbone [1-4]. The driving force for the associating process is the interaction between the hydrophobic segments in order to minimize their unfavourable exposure to water.

Vesicles are hollow spherical structures that can be formed by self-assembly of surfactants, lipids, or block copolymers in aqueous solution [5-9]. They have long been a scientific curiosity because of their structural resemblance to primitive biological cells. More importantly, vesicles are of technological interest for applications ranging from drug delivery and controlled release to bioseparations and sensing [6]. Many of these applications rely

\footnotetext{
* Corresponding author. Tel.: +00351964563851.

E-mail address: Tiago.santos@ucd.ie (T. dos Santos).
}

upon the ability of vesicles to entrap desired chemicals in their interior and thereafter release these chemicals to the external medium in a controlled manner.

The existence of thermodynamically stable vesicle phases, like in the case of catanionic mixtures, has been extensively debated in the literature [10-12]. On the other hand the classical phospholipids vesicles are normally formed by sonicating lamellar dispersions in the two-phase region with a lamellar phase in equilibrium with excess water. These vesicles are generally considered to be metastable. The $\mathrm{C}_{12} \mathrm{E}_{4}$ non-ionic vesicles used in this present work are also only kinetically stable, however they can keep their spherical topology for at least 1 week $[13,14]$.

The control of the interactions between macromolecules and surfactant lipids is the key to several applications, such as in the personal care and food industries, and in important biological functions, such as the interaction between proteins and membranes [15-18].

This investigation gives an insight into the rheological features of systems composed of associative polymers and $\mathrm{C}_{12} \mathrm{E}_{4}$ vesicles where the main driving force (hydrophobic effect) has an entropic nature. The polymer associates with the surfactant 
aggregates via the non-polar end-groups and a likely picture is a bridge formation between vesicles.

Several studies regarding the interaction between surfactant aggregates and different polymers, from non-ionic homopolymers [19-24] to HM-polyelectrolytes [25-28], have been reported. Some of these studies using charged vesicles and nonionic HM-polymers have demonstrated the independence of the association strength on the charge of the surfactant. However, the size of the surfactant aggregate was found to be very important [29].

The present study intends to give a deeper insight into the interplay of rheology properties and microstructure. First, the polymer-vesicle system will be analyzed in terms of phase map and secondly, the bridging mechanism and concentration dependence will be discussed from a rheological point of view.

\section{Experimental}

\subsection{Materials}

Tetraethylene glycol dodecyl ether $\left(\mathrm{CH}_{3}\left(\mathrm{CH}_{2}\right)_{11}\right.$ $\left.\left(\mathrm{OCH}_{2} \mathrm{CH}_{2}\right)_{4} \mathrm{OH}\right)$ was acquired from Sigma-Aldrich Co. Hydrophobically modified polyethylene glycol $\mathrm{C}_{18}\left(\mathrm{EO}_{150}\right)_{2} \mathrm{C}_{18}$ was a gift from AkzoNobel Surface Chemistry. The polymer is an end-capped hydrophobically modified copolymer, composed of $\mathrm{C}_{18}$ chains in both ends and a polar mid-block composed of 300 oxyethylene units. The average molecular weight is approximately $14,000 \mathrm{~g} / \mathrm{mol}$. Such a type of polymer shows a low polydispersity, found to be around 1.1. Both compounds were used as received. In all sample preparations, Millipore water was used.

\subsection{Sample preparation}

The polymer-surfactant mixtures were prepared by weighting the desired amounts of polymer and surfactant into small tubes equipped with a screw-cap. For the phase map determination, samples were prepared both with constant surfactant concentration (varying polymer concentration) and constant polymer concentration (varying surfactant concentration). The surfactant concentrations selected were chosen to be within the vesicle region, reported in a previously published phase diagram $[13,14]$. We assume that the polymer does not affect considerably the vesicle stability. Due to the amphiphilic character of the polymer, its dissolution in water is not trivial and cycles of vigorous hand shake, vortex mixer and centrifugation must be applied several times in order to homogenize the sample, followed by an equilibration period of at least 2 days in a stirring platform at around $25^{\circ} \mathrm{C}$.

\subsection{Rheological experiments}

Rheological measurements were performed on a Reologica Stress Tech rheometer equipped with an automatic gap setting. A plastic cone and plate geometry was employed. The sample temperature was maintained at $25 \pm 0.1^{\circ} \mathrm{C}$ by a circulating water bath. Prior to any frequency-sweep measurement a stress-sweep measurement was performed for each sample at a constant frequency of $1 \mathrm{~Hz}$ to ensure that all tests were carried out in the linear viscoelastic regime. The stress used in the linear regime was large enough to minimize instrumental noise.

Viscosity measurements, for the overlap concentration $\left(c^{*}\right)$ determination, were done in a CarriMed CSL 100 constant stress rheometer, equipped with an automatic gap setting. A $1^{\circ}$ acrylic cone and plate geometry was used. The temperature was controlled within $\pm 0.1^{\circ} \mathrm{C}$ with a Peltier control unit.

\subsection{Self-diffusion experiments}

All NMR experiments were carried out in $5 \mathrm{~mm}$ NMR tubes using a pulsed gradient spin-echo (PGSE) NMR technique on a Bruker DMX-200 spectrometer. This spectrometer operates at a ${ }^{1} \mathrm{H}$ resonance frequency of $200.13 \mathrm{MHz}$. The instrument was equipped with a Bruker DIFF-25 gradient probe driven by a Bruker BAFPA-40 unit. Here, the temperature can be varied and controlled to an accuracy of $\pm 0.2^{\circ} \mathrm{C}$. The temperature used for all measurements was $25^{\circ} \mathrm{C}$. The self-diffusion experiments were performed using the stimulated echo technique, $90^{\circ}-\tau_{1}-90^{\circ}-\tau_{2}-90^{\circ}-\tau$,-signal detection, with one pulsed field gradient of strength $g$ and duration $\delta$ in each $\tau$,-period. The time between the leading edges of the gradient pulses is $\Delta=\tau,+\tau_{2}$ and $g$ was varied in 18 equal increments to the maximum value $g_{\text {max }}=9$.

\section{Results and discussion}

\subsection{Determination of vesicle size}

Self-diffusion NMR measurements were used to calculate the vesicle radius. From the value of the relative diffusion coefficient $\left(D / D_{0}\right)$, and assuming the formation of unilamellar spherical vesicles, it is possible to determine the vesicle size according to the following equation [13]:

$R=\frac{12 l_{\mathrm{s}}\left(1-D_{\text {solv }} / D_{\text {solv }}^{0}\right)}{\phi_{\text {surf }}\left(2+D_{\text {solv }} / D_{\text {solv }}^{0}\right)}$

where $D_{\text {solv }}^{0}$ is the bulk solvent diffusion coefficient, $D_{\text {solv }}$ the solvent diffusion coefficient, $l_{\mathrm{s}}$ the volume to area ratio of surfactant and $\phi_{\text {surf }}$ is the surfactant volume fraction.

The surfactant volume fraction can be calculated by the following equation:

$\phi_{\mathrm{s}}=\frac{1}{1+\left[\left(m_{\mathrm{w}} \rho_{\mathrm{s}}\right) /\left(m_{\mathrm{s}} \rho_{\mathrm{W}}\right)\right]}$

where $m_{\mathrm{w}}$ and $m_{\mathrm{S}}$ are the mass $(\mathrm{g})$ of water and surfactant, respectively; $\rho_{\mathrm{w}}$ and $\rho_{\mathrm{S}}$ are the density $\left(\mathrm{g} / \mathrm{cm}^{3}\right)$ of the water and surfactant, respectively. The volume to area ratio of $\mathrm{C}_{12} \mathrm{E}_{4}$ is $l_{\mathrm{s}}=14.0 \AA[30,31]$.

PGSE experiments, of the water self-diffusion in the $2 \%$ of surfactant solution without polymer, were performed at $25^{\circ} \mathrm{C}$. The results are shown in Fig. 1, where echo intensity is plotted against $k$ on a semilogarithmic scale.The parameter $k$ is given 


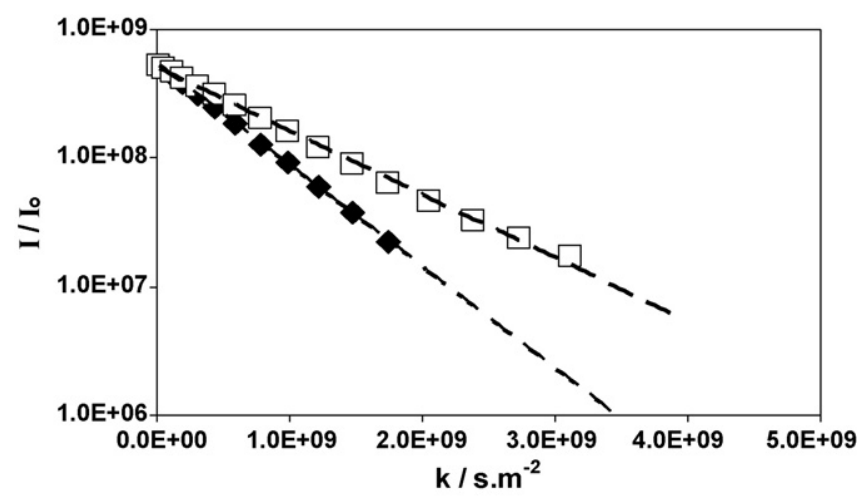

Fig. 1. Echo attenuation of water diffusion vs. $k$, at $25^{\circ} \mathrm{C}$, in pure $\mathrm{D}_{2} \mathrm{O}(\square)$ and in a sample containing $2 \mathrm{wt} \% \mathrm{C}_{12} \mathrm{E}_{4}(\diamond)$.

by the following equation:

$k=\left(\gamma^{2} g^{\prime 2} \delta^{2}\right)\left(\Delta-\left(\frac{\delta}{3}\right)\right)$

where $\gamma$ is the magnetogyric ratio of the nuclei being observed $\left(2.675 \times 10^{8} \mathrm{~s}^{-1} \mathrm{~T}^{-1}\right.$ for proton), $g^{\prime}$ the amplitude of the gradient pulse, and finally $\delta$ and $\Delta$ are the duration of the gradient pulse and the length of the diffusion time, respectively. For a better comparison, the data is also shown in Table 1.

The echo intensity decays in both cases exponentially with $k$ (giving a straight line in the semilogarithmic plot), as expected for a non-restricted diffusion. This exponential decay means that the water diffusion is characterized by a single diffusion coefficient [7]. Denoting the water self-diffusion coefficient in pure water $D_{0}$, the $D / D_{0}$ is 0.624 , which is in agreement with previous studies, using a nonsonicated solution [13]. This is consistent with the case of water being confined inside the vesicles. If we assume single shelled and monodisperse vesicles, a radius of $100 \mathrm{~nm}$ is obtained.

\subsection{Polymer-vesicle association and phase map}

The polymer adsorption onto the vesicles has a dramatic impact on the surfactant phase behaviour. Without polymer, stable vesicles are present in homogeneous bluish solutions, not optically birefringent, at the surfactant concentrations and temperature used [13].

The presence of such an associative polymer generates a different phase behaviour as compared to the one for the vesicles alone $[13,14]$. Both the phase behaviour and the rheological properties, as we will see, are strongly dependent on the polymer and surfactant concentrations. The general "diagram of states" picture displayed for the present system is mapped in

Table 1

Water self-diffusion coefficients of $2 \mathrm{wt} \%$ of surfactant aqueous solution and pure water, at $25^{\circ} \mathrm{C}$ from the pure water sample

HDO peak

$D_{\text {surfactant }}\left(\mathrm{m}^{2} \mathrm{~s}^{-1}\right)$

$1.14 \times 10^{-0.9}$

$D^{0}\left(\mathrm{~m}^{2} \mathrm{~s}^{-1}\right)$

$1.82 \times 10^{-0.9}$
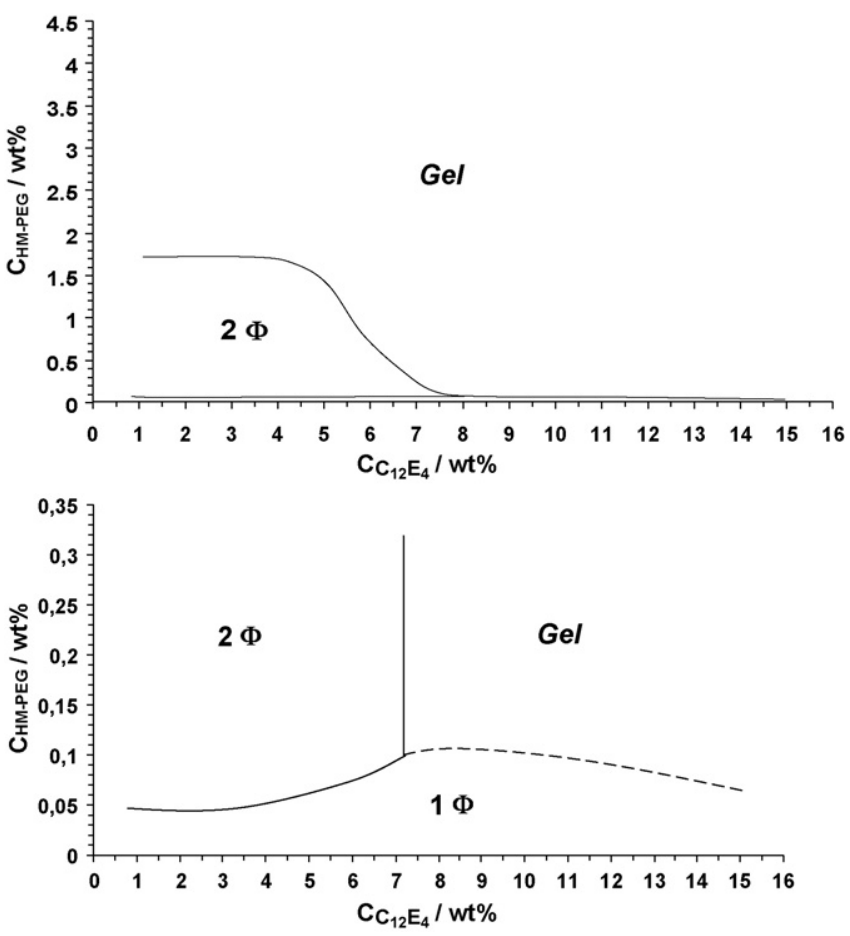

Fig. 2. Phase map of the system $\mathrm{C}_{18} \mathrm{E}_{300} \mathrm{C}_{18} / \mathrm{C}_{12} \mathrm{E}_{4}$ non-ionic vesicles (top) and a phase map expansion at lower polymer concentrations (bottom). Temperature was kept constant at $25^{\circ} \mathrm{C}$.

Fig. 2. In the monophasic region, both "liquid-like" and "gellike" behaviours are present. A phase separation composed of a concentrated phase in equilibrium with a dilute phase constitutes the scenario in the two-phase region. The map is based on the analysis of more than 120 samples. It was detected that, at lower amounts of polymer, a monophasic liquid region is detected; by increasing the polymer concentration, a biphasic region is found, just before the appearance of a gel phase region. Note that our criterion to distinguish between a "liquid" and a "gel" phase results from the rheological oscillatory measurements: the "liquid" samples always show a loss modulus, $G^{\prime \prime}$, higher than the storage modulus, $G^{\prime}$, at the investigated frequency range, while the samples denoted "gel" showed a viscoelastic, or only elastic behaviour at the entire frequency range.

A macroscopic phase separation is the optimal arrangement of the polymer-vesicle network, at specific polymer-surfactant concentrations. This phase separation explains the swelling limit of such type of networks. The water uptake is restricted (since it would involve stretching the polymer chains extensively giving a loss in conformational entropy) and therefore the excess of water goes to a bottom coexisting phase. These top phase samples, composed of polymer-surfactant network, have a bluish colour, indicating the presence of large enough particles to scatter the light perceptibly. The low density of these polymer-vesicle networks implies their location in the top phases. In similar but ionic systems [29] (associative polymers and charged surfactants) the concentrated "gel-like precipitate" was found in the bottom of the sample due to the presence of counterions "trapped" in the network. The liquid bottom phase is transparent and cloud point determinations give evidence for the presence of non-polar enti- 
ties, such as HM-PEG and/or $\mathrm{C}_{12} \mathrm{E}_{4}$. The cloud point of the liquid bottom phase is around $41{ }^{\circ} \mathrm{C}$ (for $6 \mathrm{wt} \% \mathrm{C}_{12} \mathrm{E}_{4}$ with $3 \mathrm{wt} \%$ polymer), which is close to the one found in other investigations, at $3 \mathrm{wt} \%$ of $\mathrm{HM}-\mathrm{PEG}, 37^{\circ} \mathrm{C}[19,32]$.

Comparing the present system with a related study [29], one sees that the amount of water which can be encapsulated within a network composed of vesicles and HM-PEG is higher when the vesicles are charged. This was investigated by comparing the volume fraction of two HM-PEG systems: $\mathrm{C}_{12} \mathrm{E}_{4}$ vesicles-HM-PEG and SDS/DDAB vesicles-HM-PEG. For instance, at $0.4 \mathrm{wt} \%$ of HM-PEG and $40 \mathrm{mM}$ of surfactant (i.e., $1.5 \mathrm{wt} \%$ of $\mathrm{C}_{12} \mathrm{E}_{4}$ ), the volume fraction of the gel was found to be $11 \%$ for the charged system and $5 \%$ for the nonionic system. The volume fraction of the ionic and non-ionic vesicles is, however, not the same when we use the same $C_{18}$ triblock polymer, and it is an additional parameter to control: although the $\mathrm{C}_{12} \mathrm{E}_{4}$ average radius is $100 \mathrm{~nm}$, the SDS/DDAB radius only reaches, on the conditions of the mentioned work, around $15 \mathrm{~nm}$. The larger the radius, the larger amount of water would be expected to be confined inside the vesicles. Therefore, and just considering this variable, a $\mathrm{C}_{12} \mathrm{E}_{4}-\mathrm{HM}$-PEG network with better swelling properties than the SDS/DDAB-HM-PEG network would be expected. However, the opposite situation was observed. This can be interpreted as electrostatic swelling. The latter are less polar and show only a moderate affinity with water at $25^{\circ} \mathrm{C}$. Additionally, the catanionic system
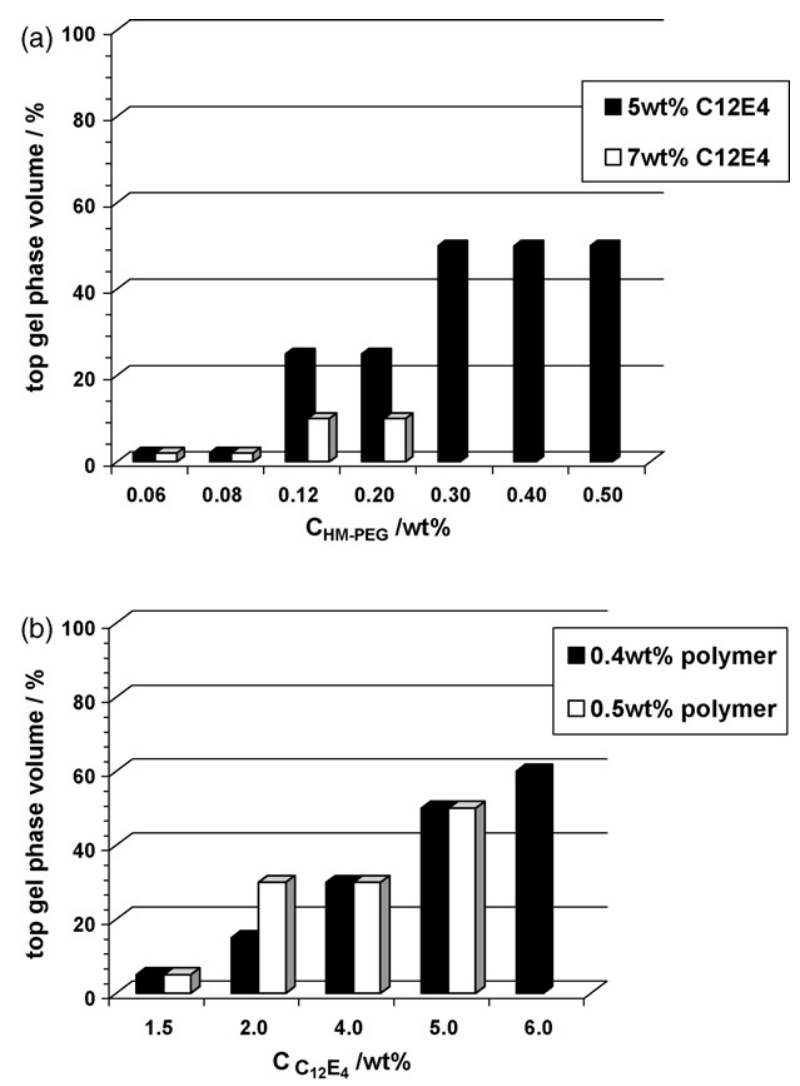

Fig. 3. Volume of the top phase (gel fraction) when (a) polymer is added to a $5 \mathrm{wt} \%$ (ם) or to a $7 \mathrm{w} \%$ ( $\square$ ) surfactant system; (b) surfactant is added to a $0.4 \%$ (ם) or to a $0.5 \%(\square)$ polymer system. has an entropic gain when the counterions are released to the medium, and extra water molecules are needed for their hydration.

In Fig. 3a and $b$ we can see that the addition of surfactant or polymer increases the network capacity to take up water since they can form new "clusters" where water is confined, increasing the volume of the top gel-like phase. Further addition of surfactant or polymer to the biphasic region stabilizes the monophasic gel region.

Such monophasic gel region was studied, particularly to understand the rheological consequences of the addition of polymer and surfactant.

\subsection{Polymer-vesicle association probed by rheology}

A strong indication of the association between the $\mathrm{C}_{18}$ triblock polymer and the non-ionic vesicles is the gel formation, even at concentrations at which the individual components only exhibit a liquid behaviour.

For a molecular understanding of this association, samples within the gel region were submitted to rheological tests. From oscillatory measurements, where a sinusoidal shear stress is applied over a range of frequencies from 0.01 to $90 \mathrm{~Hz}, G^{\prime}$ and $G^{\prime \prime}$ were determined. $G^{\prime}$ at high frequencies is proportional to the number of active links in the system, i.e., the density of polymer which forms bridges between the vesicles
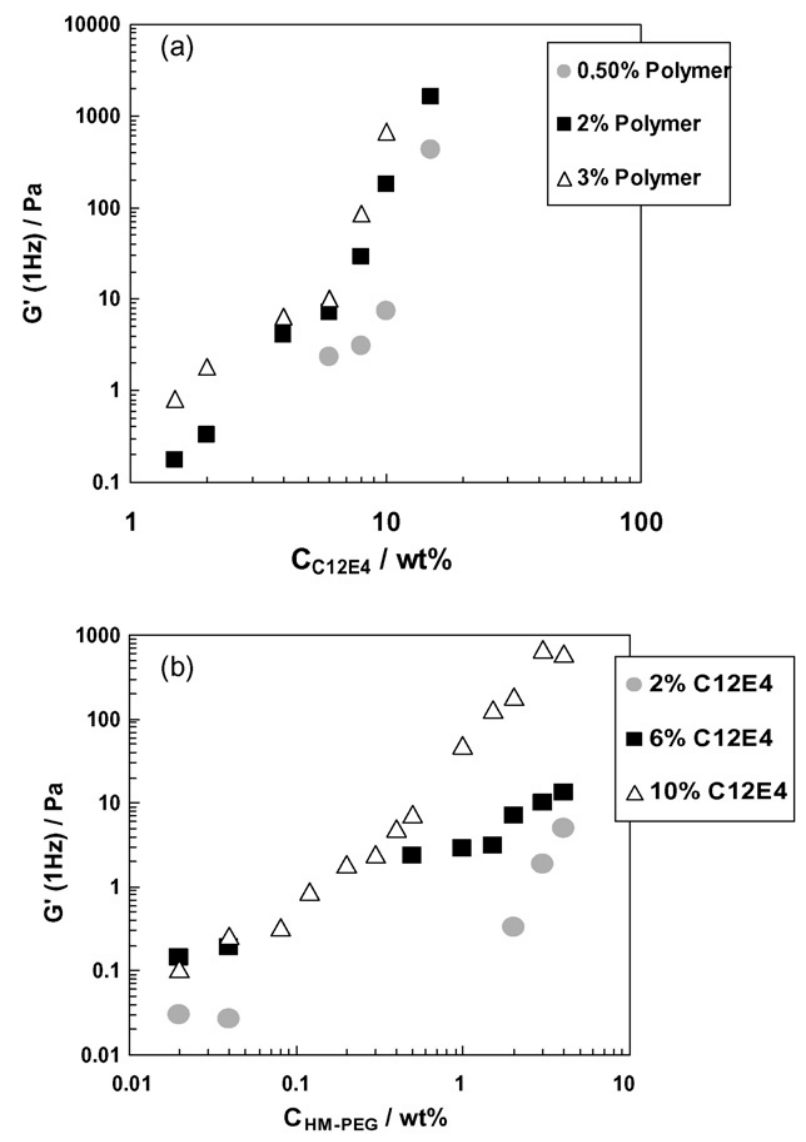

Fig. 4. Representation of $G^{\prime}$ as a function of surfactant (a) and polymer (b) concentration. 
$[22,33,34]$. The dependence of the two parameters on surfactant concentration is shown in Fig. 4. As one can see the addition of surfactant leads to an increase of $G^{\prime}$ and is expressed by an increase of the system's elasticity. Vesicles may work as crosslinking points where the polymer is anchored via its hydrophobic stickers. The addition of surfactant decreases the average distance between the vesicles and thus, the bridging effect is enhanced. This has large implications on the strength of the network, with clusters more protected against deformation.

When the stress is applied during longer times (i.e., lower frequencies), the common transition in viscoelastic systems, from solid to liquid-like behaviour, was observed. This phase transition corresponds to the time needed for the system's main relaxation, $\tau$. Many relaxation times are present in such systems; however, most of them are too fast to be perceptible (e.g. Brownian motion of the molecules). The longest $\tau$, in the current non-entangled system, corresponds to the detachment of the polymers from the vesicles. We observed that the strength of the links (seen by $\tau$ ) is enhanced and increases as the network become more consistent, due to increase of the number of polymer-vesicle connections.
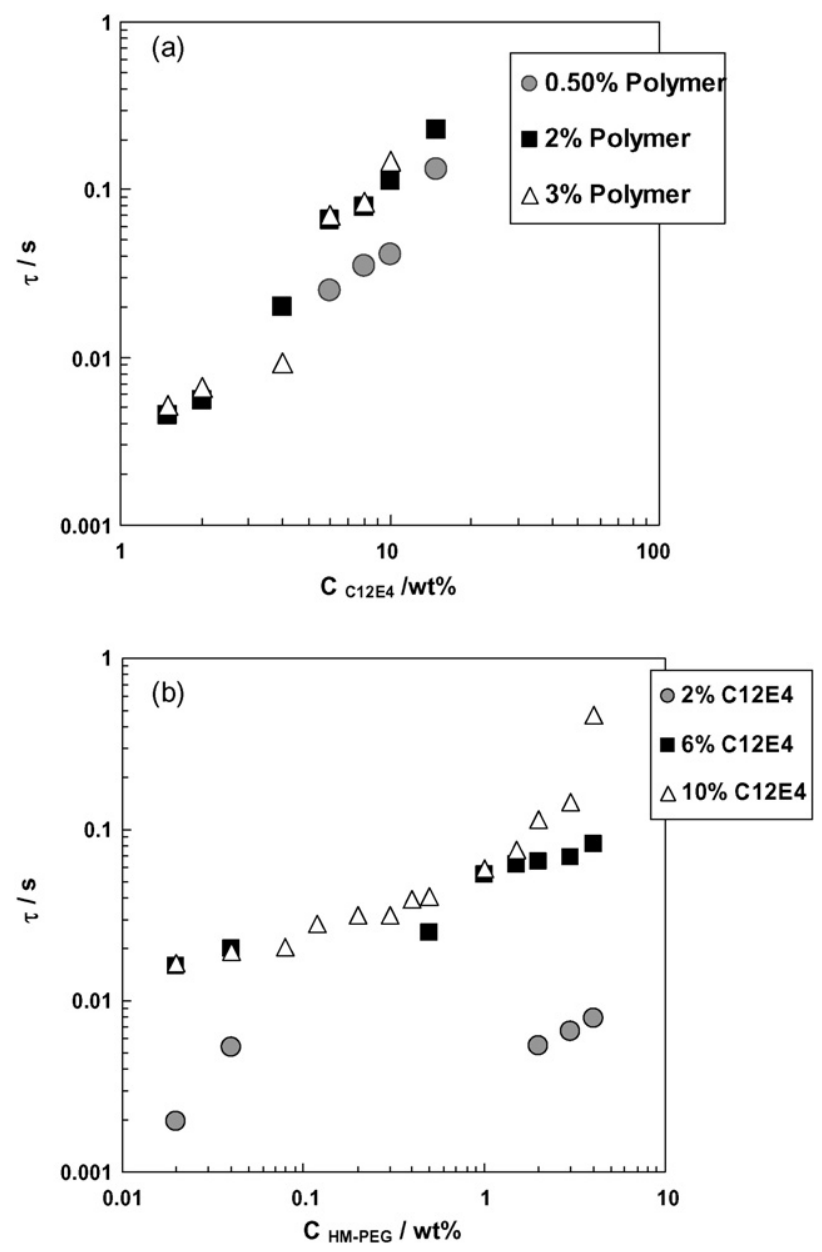

Fig. 5. Representation of the dependence of the relaxation time on surfactant (a) and polymer (b) concentration.
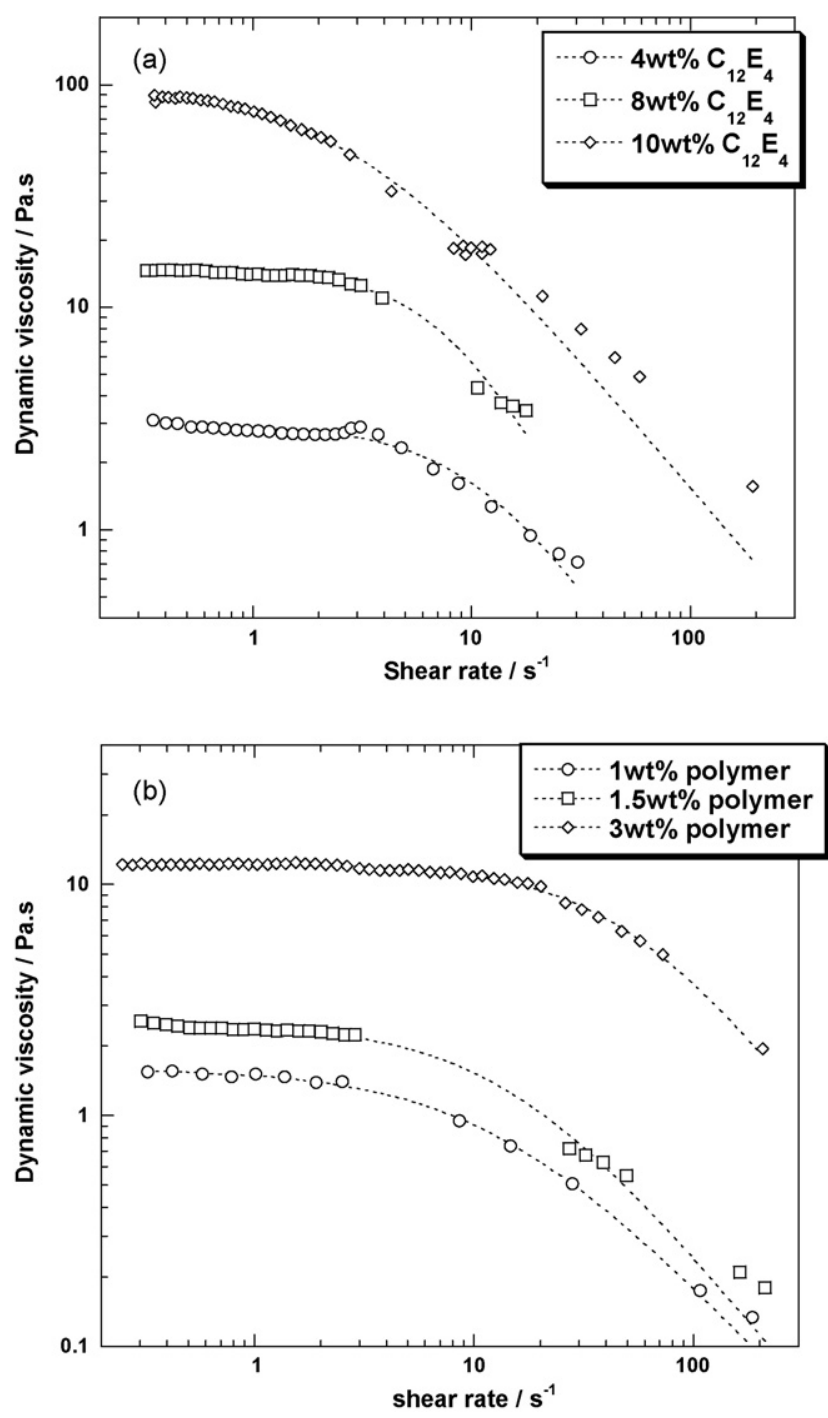

Fig. 6. Dependence of shear viscosity on shear rate. (a) Influence of surfactant concentration, at $2 \mathrm{wt} \%$ of polymer. (b) Influence of polymer concentration, at $6 \mathrm{wt} \%$ of surfactant. The dotted lines represent the Cross-equation fitting (see in the text).

The influence of polymer addition is quite strong. The dependence of $G^{\prime}$ and $\tau$ on polymer concentration is represented in Figs. 4 and 5. It is clear that the addition of polymer increases both $G^{\prime}$ and $\tau$. The addition of polymer, compared with the surfactant case seen above, induces a marked increase in the elasticity and relaxation time.

In Fig. 6, the dynamic viscosity is plotted as a function of shear rate, at both different polymer concentrations (Fig. 6a) and surfactant concentrations (Fig. 6b). In general, a Newtonian plateau at low shear rates is observed, where the viscosity is independent of the applied stress. After a critical stress, the gel structure disrupts and a shear-thinning behaviour is observed. A similar behaviour has been observed by others authors in similar systems $[19,29,35]$. We have also observed that the zero-shear viscosity always increases with the concentration of polymer and/or non-ionic vesicles in the system. Furthermore, and for a deeper understanding, the simplified Cross equation was applied and the adjusting parameters from the fitting are summarized in 
Table 2

Adjusting parameters from the Cross-equation fitting

\begin{tabular}{lllc}
\hline Sample & $\tau$ (oscillation test) (s) & $\tau$ (Cross Model) $(\mathrm{s})$ & $\eta_{0}(\mathrm{~Pa} \mathrm{~s})$ \\
\hline P2S4 & 0.020 & 0.080 & 2.95 \\
P2S8 & 0.080 & 0.120 & 14.6 \\
P2S10 & 0.11 & 0.30 & 98.1 \\
\hline
\end{tabular}

Samples are denoted as $\mathrm{P} x \mathrm{~S} y$, where $\mathrm{P}=$ polymer, $\mathrm{S}=$ surfactant, $x=$ polymer concentration and $y=$ surfactant concentration.

Table 2:

$\eta=\frac{\eta_{0}}{1+(\lambda \dot{\gamma})^{1-n}}$

In this equation (4), $\eta_{0}$ refer to the asymptotic value of viscosity at very low shear rates, $\lambda$ (or $\tau$ ) can be correlated with the structural relaxation time and $n$ is the exponent of the power law approximation in the middle shear rate region [36].

In addition, the structural relaxation time given by the Cross equation can be reasonably compared with the single relaxation time (ideal Maxwell material) obtained from the oscillatory frequency-sweep tests.

The results point out to an increase of the relaxation time and zero-shear viscosity with surfactant concentration. The influence of temperature in such non-ionic systems is currently been curried out to be presented in a forthcoming report.

\section{Conclusions}

The association between non-ionic vesicles, composed of tetraethylene glycol monododecyl ether, $\mathrm{C}_{12} \mathrm{E}_{4}$, and the associating triblock copolymer HM-poly(ethylene glycol) was investigated, mainly from a rheological point of view.

The polymer-vesicle association leads to gel-like mixtures, at sufficiently high polymer and surfactant concentrations, indicating the presence of a network. The driving force for the polymer and vesicle association has a hydrophobic nature.

A phase separation was also found, which is believed to be of an associative type, constituted by a dilute bottom phase rich in water, and a top phase rich in polymer and vesicles that associate in network domains.

The viscosity of the gels in the monophasic region is clearly influenced by the concentration of vesicle and/or polymer solutions. The same behaviour was generally observed for the storage modulus, $G^{\prime}$, and relaxation time. The volume fraction of vesicles and polymer concentration increase the global network elasticity, as well as the strength of the links. As a consequence of this enhancement of the network, the viscosity increases. Flow curves indicate that these systems are shear thinning, with disentanglement above critical shear rates. We also found a good agreement between the relaxation times found in oscillatory tests and the ones found from Cross Model applied to the flow curve.

\section{Acknowledgements}

The Colloid group in Coimbra University is supported by grants from Fundação para Ciência e Tecnologia (FCT) (refs. POCTI/QUI/35415/2000, POCTI/QUI/45344/02 and POCI/QUI/58689/2004). FEA and BM acknowledge Fundação para Ciência e Tecnologia (FCT) for research grants (refs. SFRH/BD/8168/2002 and SFRH/BD/21467/2005). Geraldine Lafitte is acknowledged for NMR measurements, Leif Karlson at Akzo Nobel Surface Chemistry, Stenungsund, for the gift of polymers, and Ulf Olsson for the stimulating discussions.

\section{References}

[1] Y. Wang, M.A. Winnik, Langmuir 6 (1990) 1437-1439.

[2] K. Holmberg, B. Jonsson, B. Kronberg, B. Lindman, Surfactants and Polymers in Aqueous Solution, 2nd ed., John Wiley, New York, 2000.

[3] L. Karlson, S. Nilsson, K. Thuresson, Colloid Polym. Sci. 277 (1999) 798-804.

[4] K. Thuresson, Solution properties of a hydrophobically modified polymer, Doctoral dissertation (thesis), Lund University, Sweden, 1996.

[5] M. Gradzielski, M. Bergmeier, H. Hoffman, M. Muller, I. Grillo, J. Phys. Chem. B 104 (2000) 11594-11597.

[6] D.D. Lasic, Liposomes, in: From Physics to Applications, Elsevier, Amsterdam, 1993.

[7] M. Gradzielski, Curr. Opin. Colloid Interf. Sci. 9 (2004) 149-153.

[8] M. Gradzielski, J. Phys. Condens. Matter 15 (2003) 655-697.

[9] H. Kunieda, K. Nakamura, T.H. Davis, F.D. Evans, Langmuir 7 (1991) 1915-1919.

[10] E.F. Marques, Association and microstructure in self-assembling catanionic systems, Doctoral dissertation (thesis), Lund University, Sweden, 2000.

[11] E. Marques, O. Regev, A. Khan, M.G. Miguel, B. Lindman, J. Phys. Chem. 102 (1995) 6746-6758.

[12] S. Rangelov, M. Almgren, Langmuir 20 (2004) 6611-6618.

[13] R. Strey, H. Kunieda, K. Nakamura, U. Olsson, Langmuir 12 (1996) 3045-3054.

[14] U. Henriksson, M. Jonstromer, U. Olsson, O. Soderman, G. Klose, J. Phys. Chem. 95 (1991) 3815-3819.

[15] B. Grasberg, P.A. Mintont, C. Delisit, H. Metzger, Proc. Natl. Acad. Sci. 83 (1986) 6258-6262.

[16] N. Taulier, C. Nicot, M. Waks, R.S. Hodges, R. Ober, W. Urbach, Biophys. J. 78 (2000) 857-865.

[17] W. Meier, J. Hotz, S. Gunther-Ausborn, Langmuir 12 (1996) 5028-5032.

[18] H.J. Lee, P.J. Gustin, T. Chen, F.G. Payne, R.S. Raghavan, Langmuir 21 (2005) 26-33.

[19] K. Thuresson, S. Nilsson, A. Kjoniksen, H. Walderhaug, B. Lindman, B. Nystrom, J. Phys. Chem. B 103 (1999) 1425-1436.

[20] P. Taboada, E. Castro, V. Mosquera, J. Phys. Chem. B 109 (2005) 23760-23770.

[21] E.F. Antunes, F.E. Marques, R. Gomes, K. Thuresson, B. Lindman, G.M. Miguel, Langmuir 20 (2004) 4647-4656.

[22] F.E. Antunes, K. Thuresson, B. Lindman, M.G. Miguel, Colloids Surf. A: Physiochem. Eng. Aspects 215 (2003) 87-100.

[23] J.R. English, H.J. Laurer, J.R. Spontak, A.S. Khan, Ind. Eng. Chem. Res 41 (2002) 6425-6435.

[24] B.H. Jorgensen, L. Coppola, K. Thuresson, U. Olsson, K. Mortensen, Langmuir 13 (1997) 4204-4218.

[25] A.S. Cartalas, I. Iliopoulos, R. Audebert, U. Olsson, Langmuir 10 (1994) 1421-1426.

[26] I. Iliopoulos, U. Olsson, J. Phys. Chem. 98 (1994) 1500-1505.

[27] B.H. Jorgensen, U. Olsson, I. Iliopoulos, K. Mortensen, Langmuir 13 (1997) 5820-5829.

[28] H.S. Ashbaugh, K. Boon, R.K. Prud'homme, Colloid Polym. Sci. 280 (2002) 783-788.

[29] B. Medronho, F. Antunes, B. Lindman, G.M. Miguel, J. Disper. Sci. Technol. 27 (2006) 83-90.

[30] T. Shimobouji, H. Matsuoka, N. Ise, H. Oikawa, Phys. Rev. A 39 (1989) 4125-4131.

[31] G. Klose, St. Eisenblatter, J. Galle, A. Islamov, U. Dietrich, Langmuir 11 (1995) 2889-2892. 
[32] P.A.M. Vieira, Studies of polymers in solution: from molecular animations to the behaviour of hydrophobically modified PEG, Diploma work, Coimbra University, Portugal, 2005.

[33] C.G. Biliaderis, Zawistowski, Cereal Chem. 67 (3) (1990) 240-246.

[34] J.D. Ferry, Viscoelastic Properties of Polymers, 3rd ed., Wiley, New York, 1980.
[35] C.K. Tam, D.R. Jenkins, A.M. Winnik, R.D. Basset, Macromolecules 31 (1998) 4149-4159.

[36] T.K.K. Goh, R.D. Haisman, H. Singh, Food Hydrocolloids 20 (2006) 176-183. 